\title{
molecules
}

ISSN 1420-3049

www.mdpi.com/journal/molecules

Article

\section{Study on the Cytotoxic Activity of Drimane Sesquiterpenes and Nordrimane Compounds against Cancer Cell Lines}

\section{Ivan Montenegro ${ }^{1,2}$, Giacomo Tomasoni ${ }^{3}$, Claudia Bosio ${ }^{3}$, Natalia Quiñones ${ }^{1}$, Alejandro Madrid ${ }^{2}$, Hector Carrasco ${ }^{4}$, Andres Olea ${ }^{4}$, Rolando Martinez ${ }^{3}$, Mauricio Cuellar ${ }^{1, *}$ and Joan Villena ${ }^{5, *}$}

1 Facultad de Farmacia, Escuela de Química y Farmacia, Universidad de Valparaíso, Av. Gran Bretaña N 1093, Valparaíso 234000, Chile; E-Mails: ivan.montenegro@uv.cl (I.M.); natuka_q@hotmail.com (N.Q.)

2 Departamento de Química, Universidad Técnica Federico Santa María, Av. España $N^{\circ} 1680$, Valparaíso 2340000, Chile; E-Mail: alejandro.madrid@usm.cl

3 Departamento de Química, Facultad de Ciencias Exactas, Universidad Andrés Bello, Quillota 910, Viña del Mar 2520000, Chile; E-Mails: volgin_4@hotmail.com (G.T.); samybrs@hotmail.com (C.B.); rmartinez@unab.cl (R.M.)

4 Facultad de Ciencias de la Salud, Universidad Autónoma de Chile, Carlos Antúnez 1920, Providencia, Santiago 7500000, Chile; E-Mails: hector.carrasco@uautonoma.cl (H.C.); andres.olea@uautonoma.cl (A.O.)

5 Centro de Investigaciones Biomédicas (CIB), Escuela de Medicina, Universidad de Valparaíso, Av. Hontaneda $N^{\circ}$ 2664, Valparaíso 234000, Chile

* Authors to whom correspondence should be addressed; E-Mails: mauricio.cuellar@uv.cl (M.C.); juan.villena@uv.cl (J.V.); Tel.: +56-032-250-8193 (M.C.).

External Editors: Jean Jacques Vanden Eynde, Annie Mayence and Tien L. Huang

Received: 23 September 2014; in revised form: 27 October 2014 / Accepted: 3 November 2014 / Published: 18 November 2014

\begin{abstract}
Twelve drimanes, including polygodial (1), isopolygodial (2), drimenol (3), confertifolin (4), and isodrimenin (5), were obtained from natural sources. Semi-synthetic derivatives 6-12 were obtained from 1 and 2, and cytotoxic activity was evaluated in vitro against cancer cell lines (HT-29, MDA-MB231, DHF, MCF-7, PC-3, DU-145, and CoN). $\mathrm{IC}_{50}$ values were determined at concentrations of $12.5-100 \mu \mathrm{M}$ of each compound for $72 \mathrm{~h}$. In addition, it was found that polygodial (1), 8, and 12 induced changes in mitochondrial membrane permeability in CoN, MCF-7, and PC-3 cells.
\end{abstract}


Keywords: cytotoxic activity; cancer cell lines; apoptosis; mitochondrial membrane permeability; caspasa-3 activity; nordrimanes; drimanes; sesquiterpenes

\section{Introduction}

The third leading cause of cancer-related deaths in women, breast cancer incidence has increased worldwide, although this may be in part due to improvements in early detection. Just as for breast cancer in women, prostate cancer is the most common non-skin malignancy in men. In the United States, for example, there were an estimated 244,000 new cases and 40,400 deaths from this cause in 1995 alone. In Chile, the risk of death from this disease has tripled in the last 40 years; the figures for 1998 indicate 1,218 deaths were recorded from prostate cancer in the country, determining a mortality rate of 16.6 per 100,000 men [1]. In Chile, prostate cancer is the second leading cause of cancer death in men, similar to developed countries. Despite the high prevalence and mortality, there are no established screening programs for early detection [2]. Due to this need, several drugs with anticancer effects have been extracted from plants in the last 20 years, many of which are particularly effective against breast cancer cells [3].

Drymis winteri Forst (Canelo) has been used in Chilean folk medicine in the treatment of inflammatory diseases [4], against tumors, uterine fibromas, malignant ulcers, and for its antifungal properties. Several sesquiterpenes have been found in Drymys species, such as polygodial (1), isopolygodial (2), drimenol (3), confertifolin (4), and isodrimenin (5) [5]. Polygodial possesses a wide range of potential biological applications, including antibacterial [6], anti-allergic and anti-inflammatory [7], and antifungal [8].

As part of a general attempt to study structure-activity relationships for unsaturated dialdehydes from natural sources, several compounds have been investigated using the Salmonella-microsome assay (strains TA 98, TA 2637, and TA 100). Polygodial and isopolygodial showed no mutagenic activity at their highest non-toxic concentration [9]. Furthermore, unsaturated sesquiterpene dialdehydes were tested for antimicrobial, cytotoxic, and mutagenic activity. Where Polygodial exhibited antibacterial and cytotoxic activity, isopolygodial was slightly less active [10].

Although the claims that $\mathbf{1}$ possesses cytotoxic activity were consistent with previous reports [11-14], contradictory reports were found in the literature regarding the cytotoxic activities of its natural analogue 2 , which was variously reported to be either active or inactive [15].

In the course of our search for bioactive compounds from natural sources, we recently reported the antifungal and antifeedant activity of naturally occurring drimanes and their semi-synthetic derivatives, including polygodial, isopolygodial, drimenol, and confertifoline [15,16]. In order to clarify the main features required for drimanes to display cytotoxic activities, we tested herein a series of 12 compounds 1-12 (most of them not previously assayed for cytotoxic activities) for antitumoral properties against a unique panel of five cancer cell lines. 


\section{Results and Discussion}

\subsection{Chemistry}

Sesquiterpenes 1-5 (Figure 1) were obtained from D. winteri [16]; compounds 6-8 were obtained from polygodial; and 9-12, from drimenol [16,17].

Figure 1. Structure of natural drimanes from bark of the $D$. winteri.

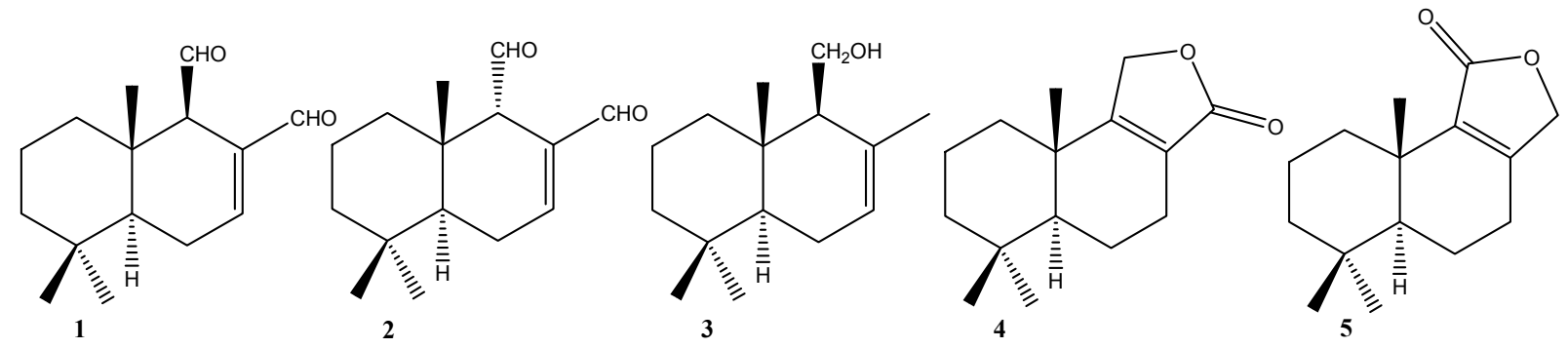

\subsubsection{Compounds Obtained from 1}

Scheme 1 shows the modifications performed on compound $\mathbf{1}$ that led to compounds $6-\mathbf{8}$. Reduction of 1 with $\mathrm{NaBH}_{4}$, in $\mathrm{MeOH}$ and at room temperature, produced $9 \beta$-drimendiol 7. Following this, when 1 was epimerized at $\mathrm{C}-9$, with $5 \% \mathrm{NaOH}$ in $\mathrm{MeOH}$ solution, and subsequently reduced with $\mathrm{NaBH}_{4}$ in $\mathrm{MeOH}$ at room temperature, $9 \alpha$-drimendiol 6 was obtained. When 1 was treated with one equivalent of ethylene glycol in benzene, in the presence of a catalytic amount of $p$-toluenesulfonic acid, monoacetal $\mathbf{8}$ was produced.

Scheme 1. Modifications performed on $\mathbf{1}$ to obtain compounds 6-8.
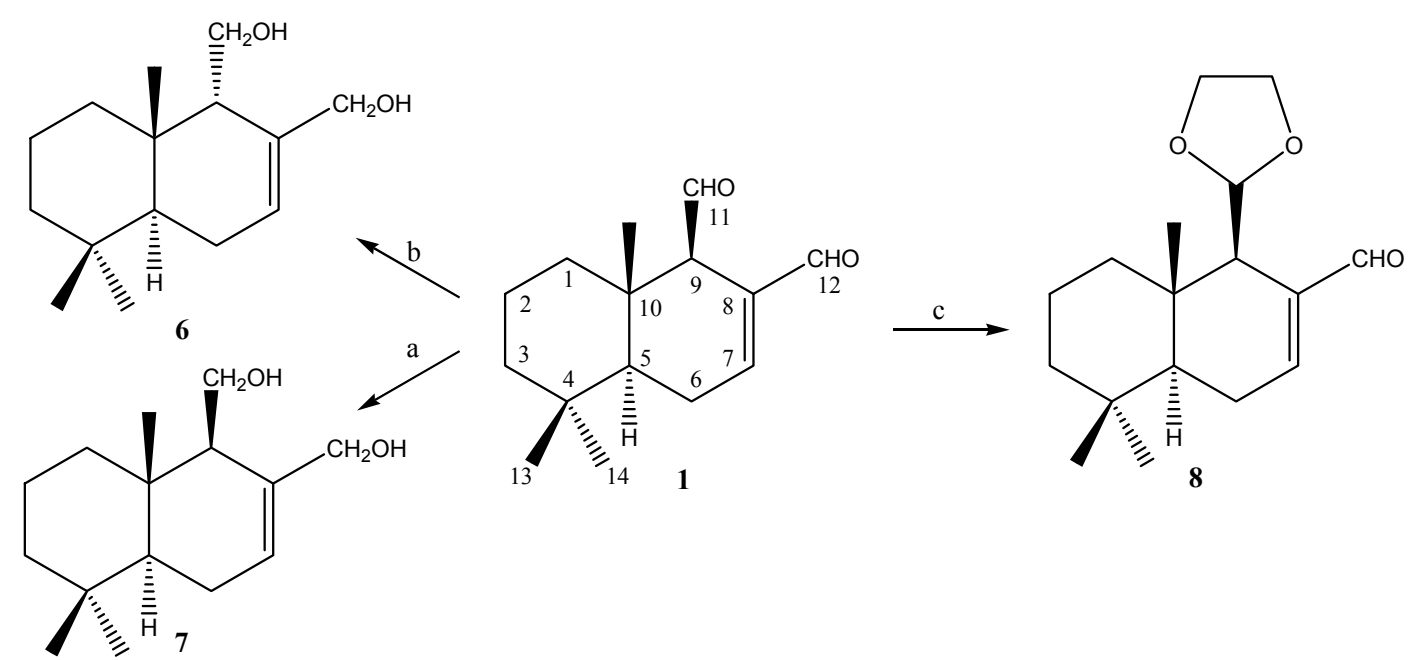

Conditions and reagents: a. $\mathrm{NaBH}_{4}$ (excess), $\mathrm{MeOH}$ rt; b. $\mathrm{KOH}, \mathrm{MeOH}(50 \%), \mathrm{NaBH}_{4}$ (excess), $\mathrm{MeOH} \mathrm{rt}$;

c. p-TosOH, ethylene glycol. 


\subsubsection{Compounds Obtained from 3}

Scheme 2 shows the modifications performed on compound 3. In this respect, we recently reported the synthesis of epoxinordrimane 9 [17]. In order to allow the synthesis of isomer 12, we proceeded to treat 3 with LDA in refluxing benzene, which allowed us to obtain 11-nordrimanic acetate $\mathbf{1 0}$.

Scheme 2. Modifications performed on $\mathbf{3}$ to obtain compounds 9-12.
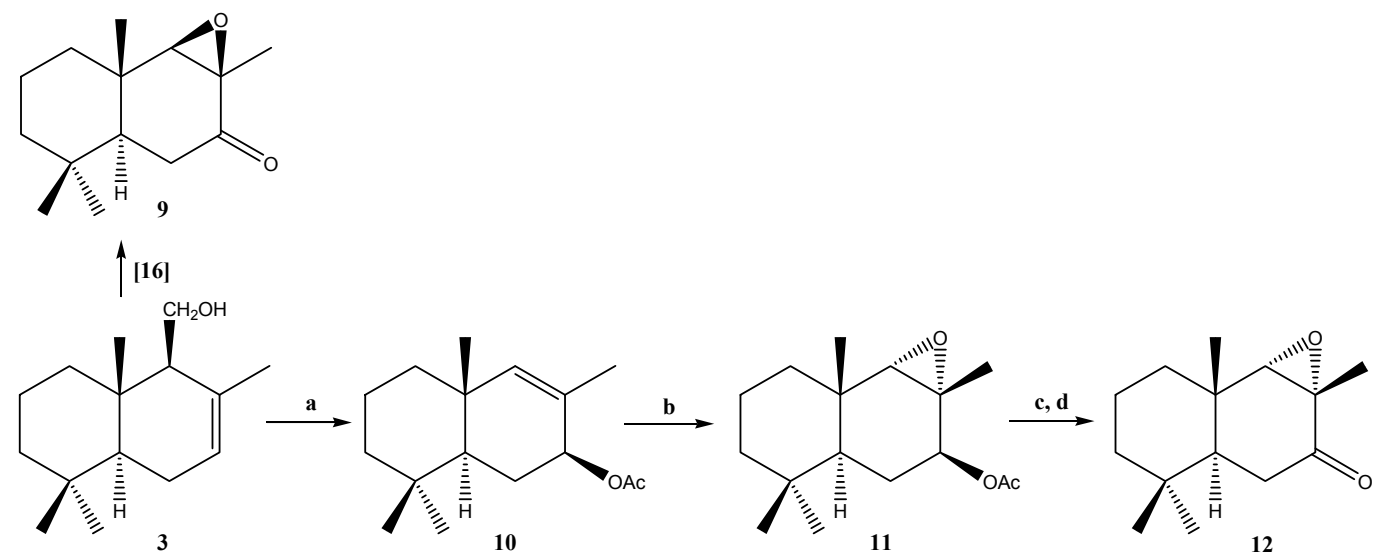

Conditions and reagents: a. LTA (lead IV acetate), $\mathrm{PhH}$, Reflux; b. m-CPBA, $\mathrm{CH}_{2} \mathrm{Cl}_{2}$, r.t, 1.5 h; c. $\mathrm{MeONa}$, $\mathrm{MeOH}, \mathrm{N}_{2}$, r.t, 2 h; d. PDC, $\mathrm{CH}_{2} \mathrm{Cl}_{2}, \mathrm{~N}_{2}$, r.t, 1.5 h.

The subsequent epoxidation of $\mathbf{1 0}$ with $m$-chloroperbenzoic acid (MCPBA) produced epoxide $\mathbf{1 1}$. Subsequent hydrolysis of $\mathbf{1 1}$ under alkaline conditions and posterior oxidation with PCC in dichloromethane lead to epoxinordrimane 12. The identities of $\mathbf{6}, 7$, and 8 were established through comparisons with previously reported data [16]. Compounds 11 and 12, which were only previously reported once, are described in detail in this work.

We recently reported a derivative series from drimenol and polygodial [15]. The assignment of the configuration in C-8 and C-9 for compound $\mathbf{1 2}$ was proposed on the basis of NOE-spectra observations. From a ${ }^{1} \mathrm{H}-1 \mathrm{D}$-NOESY experiment, when $\mathrm{CH}_{3}-12$ was selectively irradiated, only long-range interactions with $\mathrm{H}-9$ and $\mathrm{CH}_{3}-15$ were observed. In addition, when $\mathrm{H}-9$ was selectively irradiated, a long-range interaction with $\mathrm{CH}_{3}-15$ was observed. NMR experiments thus confirm the $\alpha$ spatial orientation of the epoxide ring and the stereochemistry of C-8 and C-9, as shown in Figure 2.

\subsection{Biological Results}

Previous programs designed to discover more active compounds from $D$. winteri and its derivatives, had yielded results suggesting that these compounds showed cytotoxic activity. Based on that information, and knowing that this tree has high activity potential metabolites, a growth regulatory study on cancer cell lines has been carried out.

\subsection{Viability Assay}

The cytotoxicity of compounds 1-12 was evaluated in vitro against different cancer cell lines: MCF-7 breast cancer, DU-145 and PC-3 human prostate cancer, and one non-tumor cell line, human colon epithelial cells $\mathrm{CCD} 841 \mathrm{CoN}(\mathrm{CoN})$. A colorimetric assay was set up to estimate the IC50 values, 
which represent the concentration of a drug that is required for $50 \%$ inhibition in vitro after $72 \mathrm{~h}$ of continuous exposure to the test compounds. Four serial dilutions (from 12.5 to $100 \mu \mathrm{M}$ ) for each sample were evaluated in triplicate. The IC50 obtained from these assays are shown in Table 1.

Figure 2. Bottom: ${ }^{1} \mathrm{H}-\mathrm{NMR}$ spectrum of compound 12. Top: $1 \mathrm{D}{ }^{1} \mathrm{H}$ NOESY experiment (selnogp pulse program from Bruker Library) showing the main long-range interactions between $\mathrm{CH}_{3}-12$ and $\mathrm{H}-9, \mathrm{H}-9$ and $\mathrm{CH}_{3}-15$.

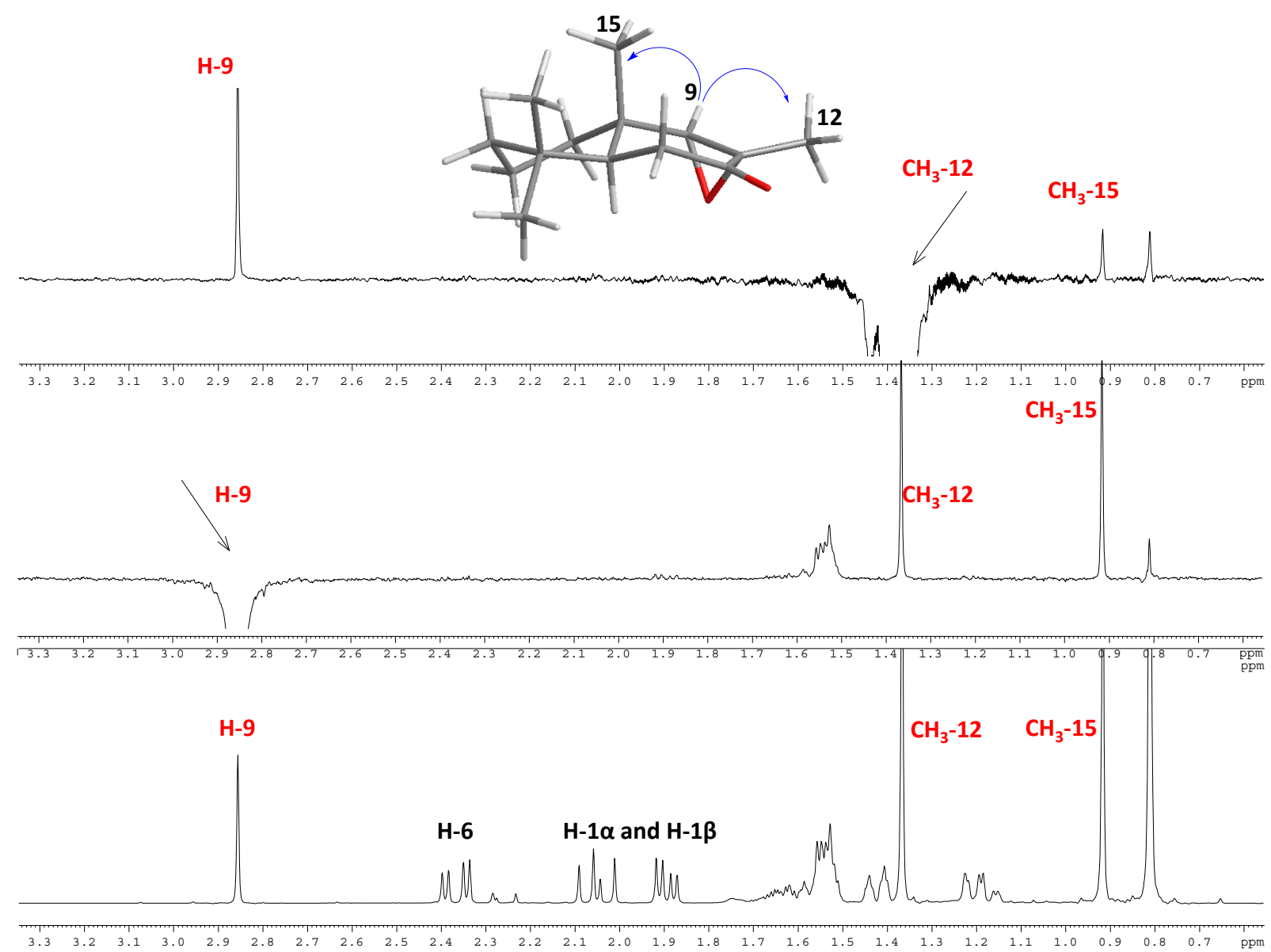

Table 1. Cytotoxicity (IC50 $\mu \mathrm{M})$ of compounds 1-12.

\begin{tabular}{cccccc}
\hline Compound & & DU145 & PC-3 & MCF-7 & CoN \\
\hline $\mathbf{1}$ & IC $_{\mathbf{5 0}}$ & $\mathbf{7 1 . 4} \pm \mathbf{8 . 5}$ & $\mathbf{8 9 . 2} \pm \mathbf{6 . 8}$ & $\mathbf{9 3 . 7} \pm \mathbf{9 . 1}$ & $>200$ \\
$\mathbf{2}$ & $\mathbf{I C}_{\mathbf{5 0}}$ & $>200$ & $>200$ & $>200$ & $>200$ \\
$\mathbf{3}$ & $\mathbf{I C}_{\mathbf{5 0}}$ & $>200$ & $>200$ & $>200$ & $>200$ \\
$\mathbf{4}$ & $\mathbf{I C}_{\mathbf{5 0}}$ & $>200$ & $>200$ & $>200$ & $>200$ \\
$\mathbf{5}$ & $\mathbf{I C}_{\mathbf{5 0}}$ & $\mathbf{9 0 . 5} \pm \mathbf{8 . 2}$ & $\mathbf{8 7 . 6} \pm \mathbf{9 . 2}$ & $>200$ & $>200$ \\
$\mathbf{6}$ & $\mathbf{I C}_{\mathbf{5 0}}$ & $>200$ & $>200$ & $>200$ & $>200$ \\
$\mathbf{7}$ & $\mathbf{I C}_{\mathbf{5 0}}$ & $>200$ & $>200$ & $>200$ & $>200$ \\
$\mathbf{8}$ & $\mathbf{I C}_{\mathbf{5 0}}$ & $\mathbf{7 0 . 6} \pm \mathbf{5 . 9}$ & $\mathbf{6 5 . 4} \pm \mathbf{5 . 5}$ & $\mathbf{9 7 . 1} \pm \mathbf{7 . 2}$ & $>200$ \\
$\mathbf{9}$ & $\mathbf{I C}_{\mathbf{5 0}}$ & $\mathbf{9 3 . 5} \pm \mathbf{6 . 7}$ & $\mathbf{9 7 . 5} \pm \mathbf{1 0 . 4}$ & $>200$ & $>200$ \\
$\mathbf{1 0}$ & $\mathbf{I C}_{\mathbf{5 0}}$ & $>200$ & $>200$ & $>200$ & $>200$ \\
$\mathbf{1 1}$ & $\mathbf{I C}_{\mathbf{5 0}}$ & $>200$ & $>200$ & $>200$ & $>200$ \\
$\mathbf{1 2}$ & $\mathbf{I C}_{\mathbf{5 0}}$ & $>200$ & $\mathbf{9 0 . 2} \pm \mathbf{8 . 8}$ & $\mathbf{8 8 . 4} \pm \mathbf{7 . 1}$ & $>200$ \\
\hline
\end{tabular}


The highest cytotoxicity values were observed for polygodial (1) and compound $\mathbf{8}$ in all cell lines tested and were more active than those of the rest of compounds. The cytotoxicity of compounds in colon epithelial cells $(\mathrm{CoN})$ is lower than in the cancer cell lines under study, indicating that the compounds are some selectivity to cancer cells.

Since compounds $\mathbf{1 , 8}$ and $\mathbf{1 2}$ had inhibitory effects on the growth of the cancer cell types tested, the effect of these compounds required study in greater detail. Moreover, the compounds $\mathbf{1}$ and $\mathbf{8}$ associated with $\alpha, \beta$-unsaturated systems and dialdehyde presence was included to probe the relevance of these groups in their biological activity on cancer cells.

First, the appearance of morphological changes in the cells treated with $50 \mu \mathrm{M}$ compound for $24 \mathrm{~h}$ was analyzed. Direct observation, using a phase contrast microscope, revealed that the morphologies of PC-3, MCF-7 and CoN cells were severely distorted and cells became rounded after treatment with compounds $\mathbf{1}, \mathbf{8}$, and 12. Moreover, the cells showed a reduction in number, indicating an increasing progression toward cell death. The control-treated cells (1\% ethanol) displayed normal and healthy shapes (Figure 3).

Figure 3. Effect of tested compounds on the morphologies of CoN, MCF-7 and PC-3 cells. Images obtained with an inverted phase contrast microscope $(200 \times)$ after 24 h exposure of the cells to $50 \mu \mathrm{M}$ of compounds $\mathbf{1}, \mathbf{8}$, and 12. (1\% ethanol) and similar to control positive (5-fluorouracil, 5-FU).
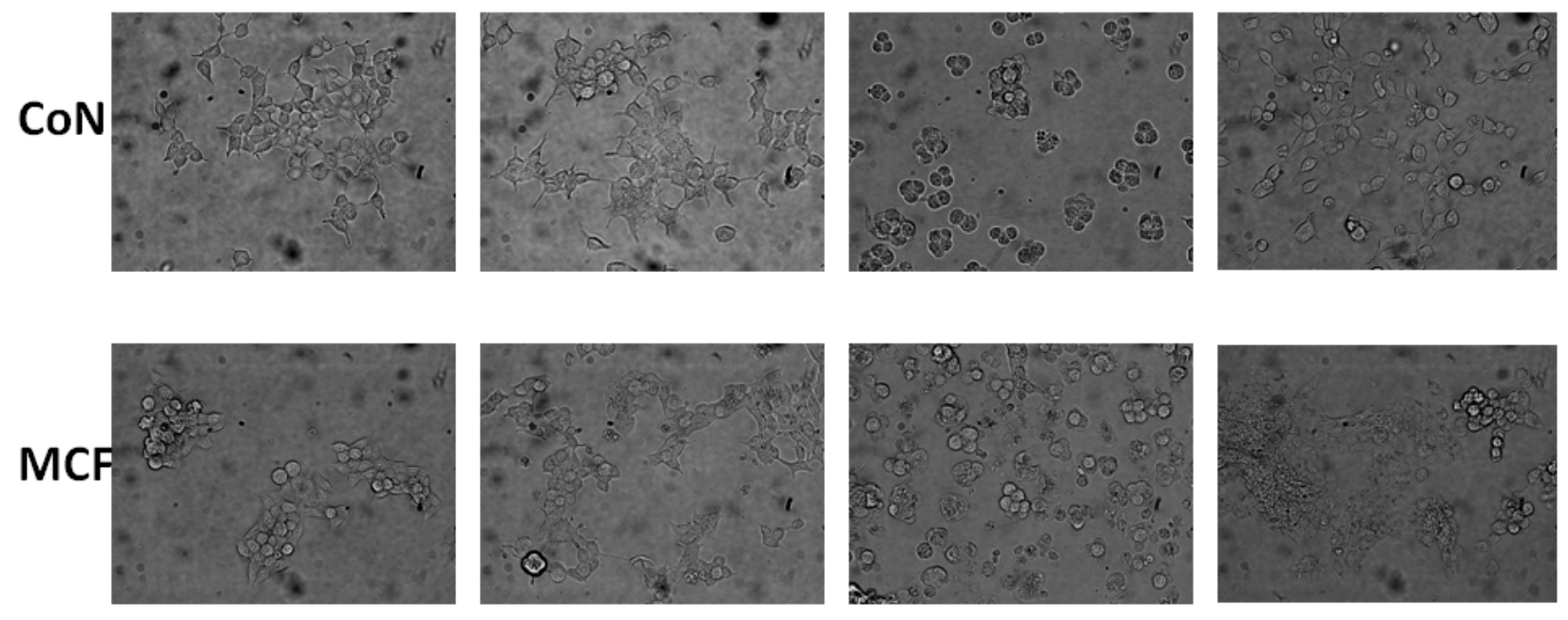

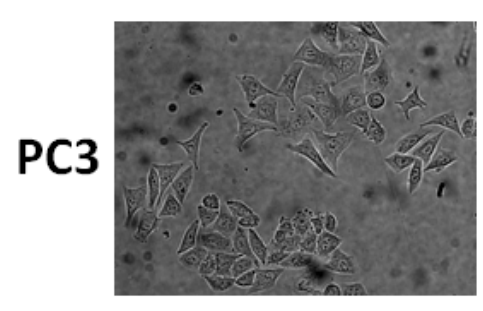

Ctrl

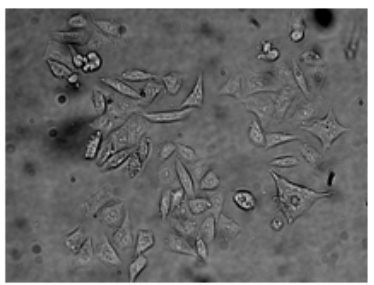

Comp.12

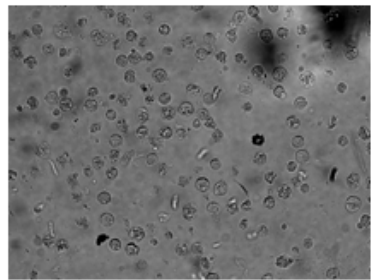

Comp. 1

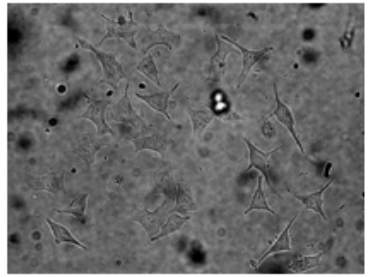

Comp. 8

To elucidate whether the compounds reduced cell viability in the cell lines tested by inducing apoptosis (MCF-7, PC-3 and CoN cells), cells treated with each active compound 1, 8 and 12 were examined after Hoechst 33342 staining. Nuclear changes in PC-3, MCF-7 and CoN cells were observed under a 
fluorescence microscope (200×, Figure 4). After exposure to the compounds for $24 \mathrm{~h}$, cells treated with compounds $\mathbf{1}$ and $\mathbf{8}$ significantly showed chromatin condensation and karyopyknosis compared to control cells (1\% ethanol) and similar to control positive (5-fluorouracil, 5-FU) which are typical characteristics of apoptotic process [18].

Figure 4. Effect of compounds $\mathbf{1 , 8}$ and 12 on chromatin condensation and fragmentation. CoN, MCF-7 and PC-3 cells treated with one of the compounds were stained with Hoechst $33342(200 \times)$. Representative photographs presented here show nuclear morphologic changes observed by fluorescent microscopy of the treated cells. As positive control, 5-fluorouracyl $(25 \mu \mathrm{M})$ was used.
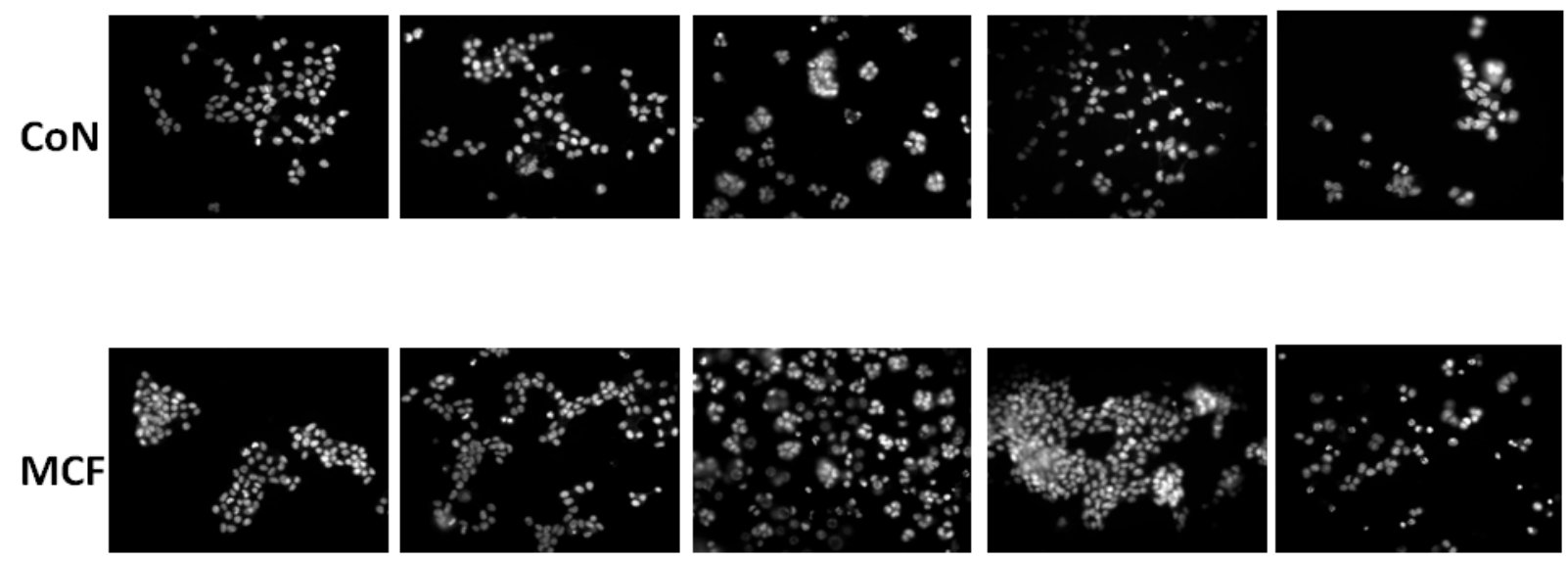

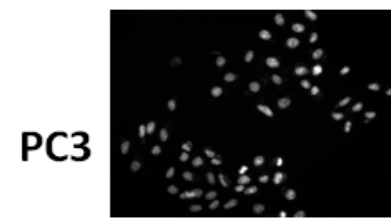

Ctrl

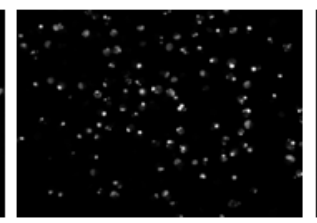

Comp. 12

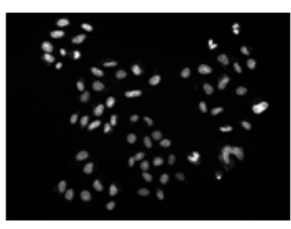

Comp. 1

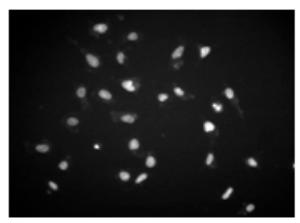

Comp. 8

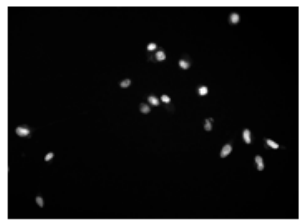

5-FU

Mitochondria play a crucial role in the apoptotic cascade by serving as a convergent center of apoptotic signals originating from both the extrinsic and intrinsic pathways [19]. Changes induced in the mitochondrial membrane potential (MMP) have been reported previously to represent a determinant in the execution of cell death [20]. As polygodial has been described as an inhibitor of mitochondrial ATPase and also as nonionic surfactant [21-23]. This effect may be determinant in cell death, we has analyzed the effect of compounds $\mathbf{1 ,} 8$ and $\mathbf{1 2}$ on the mitochondrial membrane potential using flow cytometry with rhodamine 123 stain [24]. As shown in Table 2, the percentage of rhodamine 123 stained-cells were $111.6 \% \pm 8.9 \%, 77.0 \% \pm 6.5 \%$ and $49.1 \% \pm 6.6 \%$ in the CoN, MCF-7 and PC-3, respectively, after treatment with compound $1(50 \mu \mathrm{M})$; and $96.0 \% \pm 7.6 \%, 93.3 \% \pm 7.1 \%$ and $116.2 \pm 18.8$ respectively, after treatment with compound $\mathbf{8}$, as compared to $90 \%-95 \%$ in the control cells ( $1 \%$ ethanol). Similar differences were observed after treatments with compounds $\mathbf{1}$ and 8 at $100 \mu \mathrm{M}$.

Table 2 shows that compound $\mathbf{1}$ increased mitochondrial membrane permeability in cancer cells with a greater effect than compounds 8 and 12. Moreover the compounds have a weaker effect on mitochondrial membrane potential for the epithelial colon cells, CoN, than in cancer cell lines. Thus, 
compounds $\mathbf{1}$ and $\mathbf{8}$ induced loss of mitochondrial membrane potential, correlated with increased cell death (see Table 2). However, compound $\mathbf{8}$ has a weaker effect than compound 1, indicating that the aldehyde group may be important in the loss of mitochondrial membrane potential. Moreover, compound 12 did not change the mitochondrial membrane potential when compared to control cells.

Table 2. Compounds 1, 8 and 12 treatment-induced changes in the mitochondrial membrane permeabilities for CoN, MCF-7 and PC-3 cells. The cells were stained with rhodamine 123, and then analyzed by flow cytometry. The table shows the percentage values of rhodamine 123 stained cells treated without or with compounds $\mathbf{1 , 8}$ and $12(50-100 \mu \mathrm{M})$ for the different cell lines $(* p<0.05$ or $* * p<0.001 v s$. control treated cells, assigned $100 \%)$. As positive control, FCCP $(10 \mu \mathrm{M})$ was used.

\begin{tabular}{ccccccc}
\hline & \multicolumn{2}{c}{ CoN } & \multicolumn{2}{c}{ MCF-7 } & \multicolumn{2}{c}{ PC-3 } \\
\hline & $\mathbf{5 0} \boldsymbol{\mu M}$ & $\mathbf{1 0 0} \boldsymbol{\mu} \mathbf{M}$ & $\mathbf{5 0} \boldsymbol{\mu M}$ & $\mathbf{1 0 0} \boldsymbol{\mu M}$ & $\mathbf{5 0} \boldsymbol{\mu M}$ & $\mathbf{1 0 0} \boldsymbol{\mu M}$ \\
\hline Comp. 12 & $102.6 \pm 9.1$ & $124.9 \pm 9.7$ & $111.4 \pm 8.9$ & $111.3 \pm 9.8$ & $139.1 \pm 12.4$ & $149.3 \pm 21.3$ \\
Comp. 8 & $96.0 \pm 7.6$ & $52.3 \pm 8.4 *$ & $93.3 \pm 7.1$ & $72.3 \pm 6.4 *$ & $136.2 \pm 18.5$ & $92.8 \pm 10.4$ \\
Comp. 1 & $49.1 \pm 6.6 *$ & $11.8 \pm 2.9 * *$ & $77.0 \pm 6.5 *$ & $19.4 \pm 1.5 * *$ & $111.6 \pm 8.9$ & $11.9 \pm 2.4 * *$ \\
Control & $93.2 \pm 8.7$ & & $95.8 \pm 9.9$ & & $98.1 \pm 10.5$ & \\
FCCP & & $6.8 \pm 1.3 * *$ & & $7.4 \pm 0.4 * *$ & & $5.2 \pm 0.4 * *$ \\
\hline
\end{tabular}

Figure 5. Effect of compounds 1, 8, and 12 on caspase-3 activity of MCF-7 (black), CoN (white) and PC-3 (grey) cells. Cells were exposed to compounds at $50 \mu \mathrm{M}$ for different times. Values are mean \pm S.D. $(n=3)$. All data are reported as the percentage change in comparison with the vehicle-treated cells ( $1 \%$ ethanol), which were arbitrarily assigned $100 \%$. \# $p<0.05$, significantly different from the vehicle-treated cells $(1 \%$ ethanol in medium, that is, compound concentration $=0$ ) and $* p<0.001$, significantly different from the vehicle-treated cells.

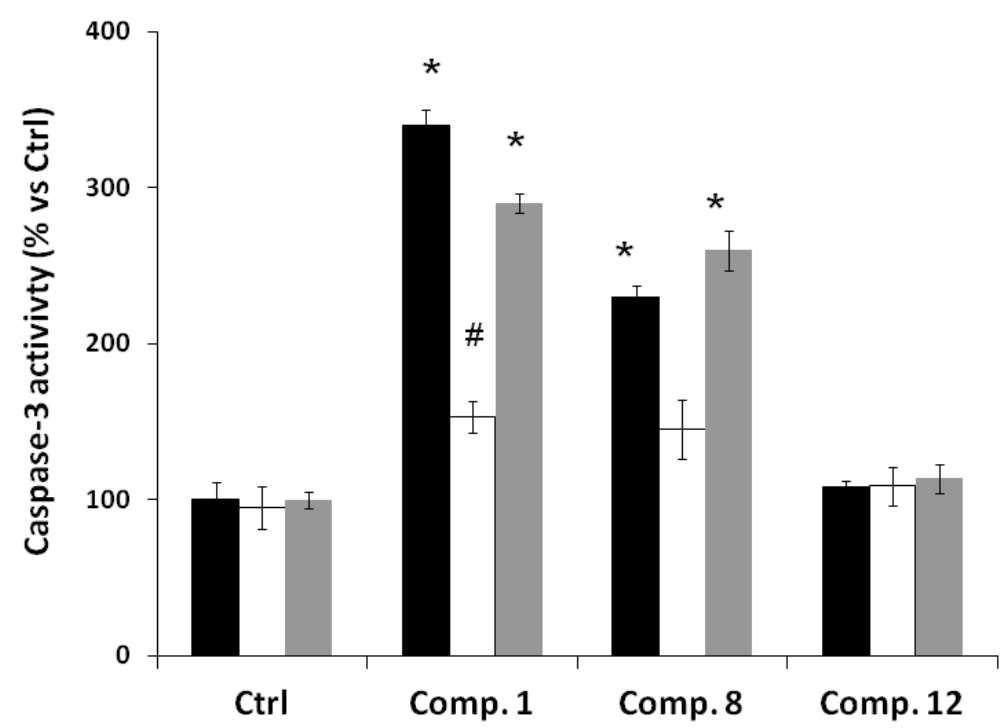

Depletion of mitochondrial membrane potential leads to the release of apoptogenic factors, such as cytochrome $\mathrm{c}$ in the apoptotic cascade, and activation of caspases $[25,26]$. Then we investigated the effects of our compounds on caspase activity. We focused on caspase-3, which is a main executor of apoptosis playing a central role in its biological processing. We analyzed the effect of treatment with 
the compound 1, 8 and 12 on caspase-3 activation in normal and cancer cells. As shown in Figure 5, the activation of caspase- 3 in cells exposed to compounds $\mathbf{1}$ and $\mathbf{8}$ is increased versus control-treated cells (1\% ethanol). Compound 1 increased the activity of caspase-3 by $3.4 \pm 0.1,1.4 \pm 0.1$ and $2.8 \pm 0.1$ times versus control cells in MCF-7 (black), CoN (white) and PC-3 (gray) cells, respectively. Compound 8 increased the activity of caspase 3 by $2.4 \pm 0.1,1.3 \pm 0.2$ and $2.7 \pm 0.1$ times versus control cells in MCF-7 (black), CoN (white) and PC-3 (gray) cells, respectively. Finally, compound 12 did not change the activity of caspase- 3 when compared with control cells.

\section{Experimental Section}

\subsection{Spectroscopic Analysis}

IR spectra were recorded as thin films on a Nicolet 6700 FT-IR spectrometer (Thermo Scientific, San Jose, CA, USA). Frequencies are reported in $\mathrm{cm}^{-1}$. ESI-MS/MS data was collected using a high-resolution hybrid quadrupole (Q) and orthogonal time-of-flight (TOF) mass spectrometer (Micromass Q-Tof, Manchester, UK) with constant nebulizer temperature of $80^{\circ} \mathrm{C}$. The ESI source and the mass spectrometer were operated in a negative ion mode, and the cone and extractor potentials were of $10 \mathrm{eV}$, with a scan range of $m / z \quad 100-500$. The band infused into the ESI source at flow rates of $5 \mu \mathrm{L} \cdot \mathrm{min}^{-1}$ was dissolved in acetonitrile ion-induced dissociation (CID) with argon in the collision chamber. The values expressed are average mass and correspond to the $[\mathrm{M}-\mathrm{H}]$ (University of Talca). ${ }^{1} \mathrm{H},{ }^{13} \mathrm{C},{ }^{13} \mathrm{C}$ DEPT-135, sel. gs $1 \mathrm{D}{ }^{1} \mathrm{H}$ NOESY, gs 2D HSQC, and $g s$ 2D HMBC spectra were recorded in $\mathrm{CDCl}_{3}$ solutions and are referenced to the residual peaks of $\mathrm{CHCl}_{3}$ at $\delta=7.26 \mathrm{ppm}$ and $\delta=77.0 \mathrm{ppm}$ for ${ }^{1} \mathrm{H}$ and ${ }^{13} \mathrm{C}$, respectively, on a Bruker Avance 400 Digital NMR spectrometer (Bruker, Rheinstetten, Germany), operating at 400.1 MHz for ${ }^{1} \mathrm{H}$ and $100.6 \mathrm{MHz}$ for ${ }^{13} \mathrm{C}$. Silica gel (200-300 mesh, Merck, Santiago, Chile) was used for column chromatography (C.C.) and HF-254 silica gel plates were used for TLC. TLC spots were detected by heating after spraying with $25 \% \mathrm{H}_{2} \mathrm{SO}_{4}$ in $\mathrm{H}_{2} \mathrm{O}$.

\subsection{Plant Material}

The stem bark of $D$. winteri adult trees was collected from the Malleco Province (Chile), in February 2010. A voucher specimen ( $\left.\mathrm{N}^{\circ} \mathrm{Dw}-10113\right)$ was deposited at the Herbarium of the Natural Products Laboratory, "Dr. Herbert Appel A.”, Department of Chemistry, Universidad Técnica Federico Santa María, Valparaíso, Chile. Fresh bark was carefully washed with abundant distilled water to remove any residue. Afterwards, it was dried in an oven at $35^{\circ} \mathrm{C}$ to a constant weight. Once dried, it was stored in hermetically sealed plastic containers at $4{ }^{\circ} \mathrm{C}$.

\subsection{Isolation of Natural Compounds $\mathbf{1}-\mathbf{4}$}

The natural drimanes polygodial (1), isopolygodial (2), drimenol (3), confertifolin (4), and isodrimenin (5) (Figure 1) were isolated from the dichloromethane extract of $D$. winteri (Winteraceae) bark. The extraction methodology and isolation of pure compounds was performed according to reported procedures [27,28]. Compounds $\mathbf{1}-\mathbf{5}$ were identified by melting point, optical rotation, and spectroscopic data, including ${ }^{1} \mathrm{H}$ - and ${ }^{13} \mathrm{C}-\mathrm{NMR}$ and comparisons with data reported in the literature $[16,28,29]$. 


\subsection{Preparation of Polygodial Derivatives 6-8 and Drimenol Derivatives 9-12}

Compounds 6-8 were synthesized by treating polygodial (1) using different protocols reported in the literature [16,30,31]. Compounds 9-12 were synthesized by treating drimenol (3) using different protocols reported in the literature [15,28,29]. The synthesis of compounds $\mathbf{1 1}$ and $\mathbf{1 2}$ is described below:

Synthesis of $(5 S, 7 S, 8 R, 9 S, 10 S)-4,4,8 \beta, 10 \beta$-tetramethyldecahydronaphtho[8,9- $\alpha$ ]oxiren-7-yl acetate (11) from 10. Compound $10(1.0 \mathrm{~g}, 3.99 \mathrm{mmol})$ was treated in $\mathrm{CH}_{2} \mathrm{Cl}_{2}(30 \mathrm{~mL})$ with $m$-CPBA $(0.94 \mathrm{~g}$, $5.4 \mathrm{mmol}$ ). Then the mixture was stirred for $1 \mathrm{~h}$ at room temperature. After this, the solution was taken up in $\mathrm{CH}_{2} \mathrm{Cl}_{2}(20 \mathrm{~mL})$ and then washed with saturated $\mathrm{NaHCO}_{3}$ solution. Later, the organic layer was dried over $\mathrm{MgSO}_{4}$, filtered, and evaporated. Then it was absorbed on silica, and subjected to C.C. eluting with mixtures of petroleum ether/EtOAc of increasing polarity $(20.0: 0.0 \rightarrow 18.0: 2.0)$ to give a white solid identified as epoxide $11(0.74 \mathrm{~g}, 68.0 \%$. $)$; m.p: $68.5-70.0{ }^{\circ} \mathrm{C} .[\alpha]_{D}^{17}=+0.36^{\circ}(\mathrm{c}=14.58$, $\mathrm{CHCl}_{3}$ ). IR ( $\mathrm{cm}^{-1}$ ): 1779; 1452; 1254. ${ }^{1} \mathrm{H}-\mathrm{NMR}: 5.05$ (t, 1H, J=8.8 Hz, H-7); 2.52 (s, 1H, H-9); 2.09

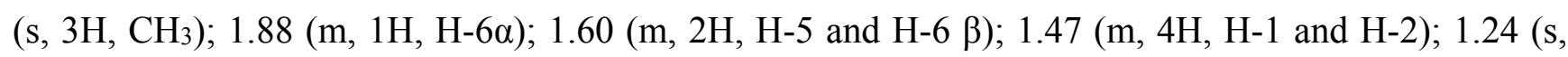
3H, H-11); 1.18 (m, 2H, H-3); 1.06 (s, 3H, H-14); 0.84 (s, 3H, H-12); 0.79 (s, 3H, H-13). ${ }^{13} \mathrm{C}-\mathrm{NMR}$ : 170.4.2 ( $\mathrm{CH}_{3} \underline{\mathrm{CO}}$ ); 71.5 (C-7); 70.7 (C-9); 58.9 (C-8); 4154 (C-5); 39.9 (C-3); 36.3 (C-1); 34.5 (C-5); 33.0 (C-10); 32.5 (C-13); 25.0 (C-12); $\left(\mathrm{CH}_{3} \mathrm{CO}\right) ; 21.1$ (C-2); 19.5 (C-14); 18.2 (C-11). HREIMS: $\mathrm{M}+\mathrm{H}$ ion $m / z 267.1882$ (calcd. for $\mathrm{C}_{16} \mathrm{H}_{26} \mathrm{O}_{3}: 266.3819$ ).

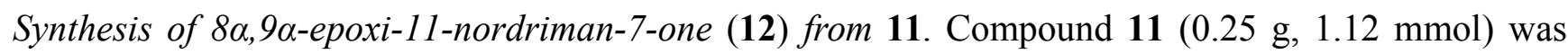
treated with a solution of $\mathrm{Na}_{2} \mathrm{CO}_{3}$ in $\mathrm{MeOH}(50 \mathrm{~mL}, 15 \%)$. Then the mixture was oxidized with PDC (1 $\mathrm{g}, 2.66 \mathrm{mmol}$ ) and was stirred for $1.5 \mathrm{~h}$ at room temperature. After this, the solution was taken up in $\mathrm{CH}_{2} \mathrm{Cl}_{2}(20 \mathrm{~mL})$ and washed with saturated $\mathrm{NaHCO}_{3}$ solution. The organic layer was dried over $\mathrm{MgSO}_{4}$, filtered, and evaporated. Then, it was absorbed on silica, and subjected to column chromatography eluting with mixtures of petroleum ether/EtOAc of increasing polarity $(20.0: 0.0 \rightarrow 18.0: 2.0)$ to give a white solid identified as epoxide 12 (0.10 g; 0.26 mmol, 40.0\%), m.p: $38-39{ }^{\circ} \mathrm{C} .[\alpha]_{D}^{17}=-0.16^{\circ}\left(\mathrm{c}=2.0, \mathrm{CHCl}_{3}\right)$. IR $\left(\mathrm{cm}^{-1}\right)$ : 1706, 1464, 1117. ${ }^{1} \mathrm{H}-\mathrm{NMR}: 2.86(\mathrm{~s}, 1 \mathrm{H}, \mathrm{H}-9)$; 2.37 (dd, 2H, H-6); 2.05 (m, 2H, H-1); 1.89 (dd, 3H, H-2 and H-5 ), 1.34 (s, 3H, H-11); 0.92 (s, 3H, H14); 0.85 (s, 3H, H-12); 0.81 (s, 3H, H-13). ${ }^{13} \mathrm{C}-\mathrm{NMR}: 207.5$ (C-7); 71.4 (C-9); 60.3 (C-8); 41.1 (C10); 41.0 (C-3); 36.4 (C-5); 34.9 (C-4); 34.4 (C-2); 32.7 (C-13); 32.1 (C-12); 20.0 (C-1); 18.3 (C-6); 17.0 (C-14); 15.5 (C-12). HREIMS: $\mathrm{M}+\mathrm{H}$ ion $m / z 223,1618$ (calcd. for $\mathrm{C}_{14} \mathrm{H}_{22} \mathrm{O}_{2}$ : 222.3286).

\subsection{Cell Lines}

The experimental cell cultures were obtained from the American Type Culture Collection (Rockville, MD, USA). MCF-7 cells (breast cancer cell line), PC-3 and DU-145 (prostate cancer cell lines), and human colon epithelial cells CCD $841 \mathrm{CoN}$ (non-tumoral cell line) were grown in Dulbecco's modified Eagle's medium (DMEM) containing 10\% FCS, $100 \mathrm{U} / \mathrm{mL}$ penicillin, $100 \mu \mathrm{g} / \mathrm{mL}$ streptomycin, and $1 \mathrm{mM}$ glutamine. Cells were seeded into 96 well microtiter plates in $100 \mu \mathrm{L}$ at a plating density of $5 \times 10^{3}$ cells/well. After $24 \mathrm{~h}$ incubation at $37^{\circ} \mathrm{C}$ under a humidified $5 \% \mathrm{CO}_{2}$ atmosphere to allow cell attachment, the cells were treated with different concentrations of drugs and incubated for $72 \mathrm{~h}$ under 
the same conditions. Stock solutions of compounds were prepared in ethanol and the final concentration of this solvent was kept constant at 1\%. Control cultures received $1 \%$ ethanol alone.

\subsection{Cell Viability}

The sulforhodamine B assay was used according to the method of Skehan et al. [32]. Briefly, the cells were set up at $3 \times 10^{3}$ cells per well of a $96200 \mu \mathrm{L}$ well, flat-bottomed, microplate. Cells were incubated at $37{ }^{\circ} \mathrm{C}$ in a humidified $5 \% \mathrm{CO}_{2} / 95 \%$ air mixture and treated with the compounds at different concentrations for $72 \mathrm{~h}$. At the end of drug exposure, cells were fixed with 50\% trichloroacetic acid at $4{ }^{\circ} \mathrm{C}$. After washing with water, cells were stained with $0.1 \%$ sulforhodamine B (Sigma-Aldrich, St. Louis, MO, USA), dissolved in $1 \%$ acetic acid (50 $\mu \mathrm{L} /$ well) for $30 \mathrm{~min}$, and subsequently washed with $1 \%$ acetic acid to remove unbound stain. Protein-bound stain was solubilized with $100 \mu \mathrm{L}$ of $10 \mathrm{mM}$ unbuffered Tris base, and the cell density was determined using a fluorescence plate reader (wavelength $540 \mathrm{~nm}$ ). Values shown are the mean \pm SD of three independent experiments in triplicate. The software used to calculate the IC50 values was GraphPad (GraphPad Software, San Diego, CA, USA).

\subsection{Morphological Assessment of Cell Apoptosis}

Morphological changes in the nuclear chromatin of cells undergoing apoptosis were revealed by a nuclear fluorescent dye, Hoechst 33342. Briefly, on 24-well chamber slides, $1 \times 10^{4}$ cells $/ \mathrm{mL}$ were cultured and exposed to compounds for $24 \mathrm{~h}$. The control group was also exposed to $1 \%$ ethanol. The cells were washed twice with phosphate buffer solution, fixed with 3.7\% formaldehyde, and washed again with phosphate buffer solution. Following the addition of $1 \mu \mathrm{M}$ Hoechst 33342 (Sigma-Aldrich, Santiago, Chile), they were reacted in a dark room at room temperature for $30 \mathrm{~min}$. After washing, they were examined under an immunofluorescence microscope (IX 81 model inverted microscope, Olympus, Santiago, Chile).

\subsection{Analysis of Mitochondrial Membrane Permeability}

Rhodamine 123, a cationic, voltage-sensitive probe that reversibly accumulates in mitochondria, was used to detect changes in transmembrane mitochondrial membrane potentials. Exponentially growing cells were incubated with the compound as indicated in the figure legends. Cells were labelled with $1 \mu \mathrm{M}$ rhodamine 123 at $37{ }^{\circ} \mathrm{C}$ in cell medium for $60 \mathrm{~min}$ before terminating the experiment. Cells were detached from the plate, after washing with ice cold PBS, and the samples were analyzed by flow cytometry. Data are expressed in percentage of cells with rhodamine 123 [24].

\subsection{Caspase 3 Activity Assay}

Caspase activity was measured using a colorimetric assay [33]. Briefly, the cells exposed to compounds were collected by centrifugation at $1000 \mathrm{rpm}$ and lysed with lysis buffer $(1 \%$ Triton X-100, $0.32 \mathrm{M}$ sucrose, $5 \mathrm{mM}$ EDTA, $10 \mathrm{mM}$ Tris- $\mathrm{HCl}$, pH 8.0, $2 \mathrm{mM}$ dithiothreitol, $1 \mathrm{mM}$ PMSF, $1 \mathrm{~g} / \mathrm{mL}$ aprotinin, $1 \mathrm{mg} / \mathrm{mL}$ leupeptin). Thereafter, the lysates were transferred to wells in a 96-well microplate and were incubated with DEVD-pNA (final concentration $200 \mu \mathrm{M}$ ) specific for caspase-3/7, at $37{ }^{\circ} \mathrm{C}$ for $1 \mathrm{~h}$. The intensity of the developed color was read at $405 \mathrm{~nm}$ in a microplate reader (SpectraMax, Winooski, VT, USA). The results are expressed as percentages of the control level. 


\subsection{Statistical Analysis}

Data shown in tables are average results obtained by means of three or 10 replicates and are presented as average \pm standard errors of the mean (SEM). Data were subjected to analysis of variance (ANOVA) with significant differences between means identified by GLM procedures. Results are given in the text as probability values, with $p<0.05$ adopted as the criterion of significance. Differences between treatment means were established with a Student-Newman-Keuls (SNK) test. The EC50 values for each activity were calculated by PROBIT analysis based on percentage of mortality obtained at each concentration of the samples. $\mathrm{EC}_{50}$ is half maximal effective concentration. Complete statistical analysis was performed by means of the MicroCal Origin 6.0 statistics and graphs PC program.

\section{Conclusions}

The results show that only polygodial (1), isodrimenin (5), aldehyde 8 and compounds 9 and $\mathbf{1 2}$ had $\mathrm{IC}_{50}$ values lower than $200 \mu \mathrm{M}$ against a panel of cancer cell lines. We found that a higher concentration of polygodial (1) decreases the cell viability of DU-145 and PC-3, MCF-7. Drimenol (3) and confertifolin (4) have an effect on the cell lines at higher concentrations, but only in PC-3 did they show activity against tested cells, DU-145, at lower concentrations. Compounds $\mathbf{1}$ and $\mathbf{8}$ showed a good apoptotic response against prostate cancer cell cultures. Moreover, compounds $\mathbf{1}$ and $\mathbf{8}$ also depleted the mitochondrial membrane potential and increased caspase-3 activity. Compound $\mathbf{1 2}$ neither changed the mitochondrial membrane permeability nor the activity of caspase-3, suggesting that the presence of a dialdehyde had relevance for the apoptotic activity in the cell lines studied.

\section{Acknowledgments}

The authors thank Innova-CORFO (grant 09MCSS6683) and the DGIP of the Universidad Técnica Federico Santa María (PAC 2014 and PIIC 2013) for financial support.

\section{Author Contributions}

IM, MC and JV designed research; IM, GT, CB, AM, NQ, HC, AO and RM performed research and analyzed the data; IM, MC and JV wrote the paper. All authors read and approved the final manuscript.

\section{Conflicts of Interest}

The authors declare no conflict of interest.

\section{References}

1. Baechler, R.; Henríquez, R.; Aqueveque, X.; Martínez, M.; Soto, A. Prevalence of prostate cancer in the Seventh Region of Chile. Rev. Med. Chile 2001, 11, 1305-1310.

2. Novoa, C.; Aliaga, A.; Badilla, S.; Reyes, D. Current reality of the screening for prostate cancer. Are we carrying out the recommendations? Rev. Chil. Urol. 2013, 78, 27-31. 
3. Echiburú-Chau, C.; Alfaro-Lira, S.; Brown, N.; Salas, C.; Cuellar, M.; Santander, J.; Ogalde, J.; Rothhammer, F. The selective cytotoxicity elicited by phytochemical extract from Senecio graveolens (Asteraceae) on breast cancer cells is enhanced by hypoxia. Int. J. Oncol. 2014, 44, 1357-1364.

4. Estomba, D.; Ladio, A.; Lozada, M. Medicinal wild plant knowledge and gathering patterns in a Mapuche community from North-western Patagonia. J. Ethnopharmacol. 2006, 103, 109-119.

5. Jansen, B.J.M.; de Groot, A. Occurrence, biological activity and synthesis of drimane sesquiterpenoids. Nat. Prod. Rep. 2004, 21, 449-477.

6. Fujita, K.; Kubo, I. Multifunctional action of antifungal polygodial against Saccharomyces cerevisiae: Involvement of pyrrole formation on cell surface in antifungal action. Bioorg. Med. Chem. 2005, 13, 6742-6747.

7. Cunha, F.; Fröde, T.; Mendes, G.; Malheiros, A.; Filho, V.C.; Yunes, R.A.; Calixto, J.B. Additional evidence for the anti-inflammatory and anti-allergic properties of the sesquiterpene polygodial. Life Sci. 2001, 70, 159-169.

8. Malheiros, A.; Filho, V.C.; Schmitt, C.; Yunes, R.; Escalante, A.; Svetaz, L.; Zacchino, S.; Delle Monache, F. Antifungal activity of drimane sesquiterpenes from Drimys brasiliensis using bioassay-guided fractionation. J. Pharm. Pharm. Sci. 2005, 8, 335-339.

9. Sterner, O.; Carter, R.E.; Nilsson, L.M. Structure-activity relationships for unsaturated dialdehydes. 1. The mutagenic activity of 18 compounds in the Salmonella/microsome assay. Mutat. Res. 1987, $188,169-174$.

10. Anke, H.; Sterner, O. Comparison of the antimicrobial and cytotoxic activities of twenty unsaturated sesquiterpene dialdehydes from plants and mushrooms. Planta Med. 1991, 57, 344-346.

11. Mc Callion, R.; Cole, A.; Walker, J.; Blunt, J.; Munro, M. Antibiotic substances from New Zealand plants. Planta Med. 1982, 44, 134-138.

12. De Almeida Alves, T.; Ribeiro, F.; Kloos, H.; Zani, C. Polygodial, the fungitoxic component from the Brazilian medicinal plant Polygonum punctatum. Mem. Inst. Oswaldo Cruz 2001, 96, 831-833.

13. Taniguchi, M.; Adachi, T.; Oi, S.; Kimura, A.; Katsumura, S.; Isoe, S.; Kubo, I. Structure-activity relationship of the Warburgia sesquiterpene dialdehydes. Agric. Biol. Chem. 1984, 48, 73-78.

14. Lee, S.; Lee, J.; Lunde, C.; Kubo, I. In vitro antifungal susceptibilities of Candida albicans and other fungal pathogens to polygodial, a sesquiterpene dialdehyde. Planta Med. 1998, 65, 204-208.

15. Derita, M.; Zacchino, S. Validation of the ethnopharmacological use of Polygonum persicaria for its antifungal properties. Nat. Prod. Commun. 2011, 6, 931-933.

16. Montenegro, I.; Pino, L.; Werner, E.; Madrid, A.; Espinoza, L.; Moreno, L.; Villena, J.; Cuellar, M. Comparative Study on the larvicidal activity of drimane sesquiterpenes and nordrimane compounds against Drosophila melanogaster til-til. Molecules 2013, 18, 4192-4208.

17. Derita, M.; Montenegro, I.; Garibotto, F.; Enriz, R.; Cuellar, M.; Zacchino, S. Structural requirements for the antifungal activities of natural drimane sesquiterpenes and analogues, supported by conformational and electronic studies. Molecules 2013, 18, 2029-2051.

18. Allen, P.D.; Newland, A.C. Apoptosis detection by DNA analysis. Methods Mol. Med. 1996, 6, 207-213.

19. Fleischer, A.; Ghadiri, A.; Dessauge, A.F.; Duhamela, M.; Rebollo, M.P.; Alvarez-Franco, F.; Rebollo, A. Modulating apoptosis as a target for effective therapy. Mol. Immunol. 2006, 43, 1065-1079. 
20. Villena, J.; Madrid, A.; Montenegro, I.; Werner, E.; Cuellar, M.; Espinoza, L. Diterpenylhydroquinones from Natural ent-Labdanes Induce Apoptosis through Decreased Mitochondrial Membrane Potential. Molecules 2013, 18, 5348-5359.

21. Lunde, C.; Kubo, I. Effect of Polygodial on the Mitochondrial ATPase of Saccharomyces cerevisiae. Antimicrob. Agents Chemother. 2000, 44, 1943-1953.

22. Kubo, I.; Fujita, K.I.; Lee, S.H. Antifungal mechanism of polygodial. J. Agric. Food Chem. 2001, 49, 1607-1611.

23. Castelli, V.; Lodeyro, A.; Malheiros, A.; Zacchino, S.; Roveri, O. Inhibition of the mitochondrial ATP synthesis by polygodial, a naturally occurring dialdehyde unsaturated sesquiterpene. Biochem. Pharmacol. 2005, 70, 82-89.

24. Emaus, R.K.; Grunwald, R.; Lemaster, J.J. Rhodamine 123 as a probe of transmembrane potential in isolated rat-liver mitochondria: Spectral and metabolic properties. Biochim. Biophys. Acta 1986, 850, 436-448.

25. Kim, R.; Emi, M.; Tanabe, K. Role of mitochondria as the gardens of cell death. Cancer Chemother. Pharmacol. 2006, 57, 545-553.

26. Mattson, M.P.; Kroemer, G. Mitochondria in cell death: Novel targets for neuroprotection and cardioprotection. Trends Mol. Med. 2003, 9, 196-205.

27. Moreno-Osório, L.; Cortés, M.; Armstrong, V.; Bailén, M.; González-Coloma, A. Antifeedant activity of some polygodial derivatives. Z. Naturforsch. C 2008, 63, 215-220.

28. Derita, M.G.; Leivab, M.L.; Zacchino, S.A. Influence of plant part, season of collection and content of the main active constituent, on the antifungal properties of Polygonum acuminatum Kunth. J. Ethnopharmacol. 2009, 124, 377-383.

29. Rodríguez, B.; Zapata, N.; Medina, P.; Viñuela, E. A complete ${ }^{1} \mathrm{H}$ and ${ }^{13} \mathrm{C}-\mathrm{NMR}$ data assignment for four drimane sesquiterpenoids isolated from Drimys winteri. Magn. Reson. Chem. 2005, 43, 82-84.

30. Hueso-Rodríguez, J.; Rodríguez, B. A new and efficient route to optically active drimanes. Synthesis of $(+)$-winterin, $(+)$-confertifolin, $(+)$-isodrimenin, and $(+)$-bicyclofarnesol. Tetrahedron 1989, 45, 1567-1576.

31. Cortés, M.; Delgado, V.; Saitz, C.; Armstrong, V. Drimenol: A versatile synthon for compounds with trans-drimane skeleton. Nat. Prod. Commun. 2011, 6, 477-490.

32. Skehan, P.; Storeng, R.; Scudiero, D.; Monks, A.; McMahon, J.; Vistica, D.; Warren, J.T.; Bokesch, H.; Kenney, S.; Boyd, M.R. New colorimetric cytotoxicity assay for anticancer-drug screening. J. Natl. Cancer Inst. 1990, 82, 1107-1112.

33. Day, T.W.; Wu, C.H.; Safa, A.R. Etoposide induces protein kinase C C $\delta$ - and caspase 3 dependent apoptosis in neuroblastoma cancer cells. Mol. Pharmacol. 2009, 76, 632-640.

Sample Availability: Samples of the compounds 1-12 are available from the authors.

(C) 2014 by the authors; licensee MDPI, Basel, Switzerland. This article is an open access article distributed under the terms and conditions of the Creative Commons Attribution license (http://creativecommons.org/licenses/by/4.0/). 